\title{
CONSTRUCCIÓN DEL CONOCIMIENTO PRÁCTICO, A PARTIR DE LA PRAXIS DE UNA DOCENTE EN FORMACIÓN
}

\author{
Por: Marcela Neira ${ }^{1}$ y Análida Hernández ${ }^{2}$
}

\begin{tabular}{|l|}
\hline Recibido:27-04-2012 \\
\hline Aceptado:06-07-2012 \\
\hline
\end{tabular}

\section{Resumen}

El presente artículo aporta a la construcción del Conocimiento Práctico, por medio del análisis de una práctica reflexiva, de una docente en formación del Proyecto Curricular de Licenciatura en Biología, de la Universidad Pedagógica Nacional. Dicha reflexión, se llevó a cabo en el espacio académico Práctica Pedagógica y Didáctica Específica II.

La metodología estructurada corresponde a una perspectiva etnometodológica, un enfoque cualitativo y de carácter interpretativo. Los métodos utilizados fueron biográfico, análisis de contenido, estudio de caso y las técnicas empleadas: Bitácora y relato autobiográfico.

Las conclusiones estipuladas se relacionan con los objetivos y con la metodología; entre las que se destaca la importancia de construir el Conocimiento Práctico, a través de la descripción de la práctica pedagógica, sus respectivas reflexiones y entenderlas a la luz de las narraciones de vida del enseñante. Así mismo, para su análisis se requiere la diferenciación entre "el pensamiento real e ideal".

Palabras clave: Conocimiento profesional del profesor, conocimiento práctico, práctica reflexiva, práctica pedagógica y formación inicial.

\section{Abstract}

This paper brings contributions to the construction of practical knowledge, through the analysis of reflective practice, a teacher training Proyecto Curricular de Licenciatura en Biología, at the Universidad Pedagógica Nacional. Such consideration was carried out in the academic space academic Pedagógica y Didáctica Específica II.

The used methodology corresponds to a perspective etnometodológica, a qualitative approach and of interpretive character. The methods are biographical, analysis of content, study of case and the techniques used are the log and autobiographical.

${ }^{1}$ Egresada de Licenciatura en Biología, Universidad Pedagógica Nacional (Colombia). Integrante del Grupo de Investigación Conocimiento Profesional del Profesor de Ciencias: neira.marce@gmail.com

2 Profesora del Departamento de Biología, Universidad Pedagógica Nacional (Colombia). Coordinadora del Grupo de Investigación Conocimiento Profesional del Profesor de Ciencias: anaherpi@hotmail.com 
The stipulated conclusions are related to the targets and to the methodology. Between those that there emphasizes the importance of constructing the Practical Knowledge, across the description of the teaching practice, its respective reflections and of understanding them in view of the stories of life of the teacher. Likewise, for its analysis is needed the differentiation between "the real and ideal thought".

Key Words: Teacher professional knowledge, practical knowledge, reflexive practice, pedagogic practice and teacher development.

\section{Introducción:}

En la actualidad existe la idea, que para llevar a cabo la docencia, sólo se requiere el dominio del Conocimiento del Contenido Disciplinar (CCD); desconociendo que los maestros poseen un conocimiento particular, que les permite transformar los conocimientos inmersos en la enseñanza (Valbuena, 2007).

Ésta representación ha ocasionado que el sistema educativo conciba al maestro, como un simple agente prestador de servicios, sin un rango profesional definido y valorizado (Soto, 2006). Recientemente, ésta problemática se refleja en la reforma al Estatuto Docente Colombiano, decreto 1278 de 2002 (Artículo 7), en el cual se permite el ingreso de profesionales no formados en la educación, donde se les exige cursar un año de estudios pedagógicos (Estatuto Docente Colombiano: Decreto 1278, 2002).

Reformas de éste tipo, que subestiman la labor del enseñante, desconocen el postulado de que los maestros son "sujetos de conocimiento" (Tardif: 2004; 167), los cuales poseen unos conocimientos específicos que utilizan y generan en el ámbito de sus labores diarias. Ocasionando, que personas del común y autores como Perrenoud (2001), conciban la labor de la enseñanza como una semiprofesión.

Sin embargo, en contraposición a éste planteamiento, en la literatura se privilegia el modelo de desarrollo profesional como investigador, el cual propone la integración entre la teoría con la práctica y ubica al docente, como aquél agente que desarrolla procesos de reflexión crítica en su labor (Imbernón, 1998). Por consiguiente, dentro de éste modelo se revaloriza la práctica pedagógica, no sólo como "un espacio de aplicación de saberes provenientes de la teoría, sino también un espacio de producción de saberes específicos que proceden de esa misma práctica" (Tardif, 2004: 172).

Al tener presente lo anteriormente mencionado, frente al estatus docente no valorizado y el desconocimiento por parte de los maestros de la posesión de un conocimiento particular, que los faculta para enseñar; éste artículo pretende mostrar una investigación, que tuvo como objetivo aportar a la construcción del Conocimiento Práctico, a partir del análisis de una práctica reflexiva del espacio académico Práctica Pedagógica y Didáctica Específica II, de una docente en formación en Biología, de la Universidad Pedagógica Nacional (Neira, 2012). Cabe resaltar, que ésta investigación corresponde a un trabajo de grado, donde una de las autoras del artículo, es el mismo objeto de investigación y al mismo tiempo asumió el rol de investigadora.

Para alcanzar la meta estipulada, se sistematizó las reflexiones de la práctica docente, denominada "Desarrollo de competencias comunicativas, a partir de la implementación de una unidad didáctica en ecología y del modelo de enseñanza para la comprensión" (Neira, 
2009); posteriormente, se analizó la praxis a favor de la contribución al Conocimiento Práctico y por último, se evaluó los niveles reflexibilidad alcanzados por la docente en formación, a partir de los criterios propuestos por Viciana, Delgado \& Del Villar (1997). Estos objetivos específicos fueron estructurados, teniendo en cuenta que el conocimiento experiencial al ser explícito, conlleva a posicionar a la enseñanza como una actividad intelectual mediante la constante producción docente.

Dicho análisis no sólo contribuyó en procesos de cualificación de la docente en formación, sino también fortaleció el marco teórico del concepto de práctica reflexiva, donde se propuso algunas recomendaciones a nivel estatal y universitario, para incentivar la apropiación de la reflexión en la docencia.

Por otra parte, a continuación se presenta una breve conceptualización de algunos términos relevantes en la investigación, como lo son: Conocimiento docente, conocimiento práctico y práctica reflexiva; con el propósito de contextualizar al lector en el estudio.

Viscaíno (2008), afirma que el conocimiento docente, es una construcción social y personal, que evoluciona y se nutre de diferentes fuentes (experiencias de vida, formación académica y socialización profesional). Percibe al conocimiento desde una marcada diferenciación, entre el conocimiento teórico y el conocimiento, que el docente ha elaborado en contextos prácticos. Por tanto, propone rescatar la reflexión, como medio para articular los conocimientos y como elemento para la reconstrucción del mismo, a partir de la comprensión de la práctica pedagógica.

Dentro de ésta visión, uno de los conceptos a realzar en primer instante, es el de Conocimiento Práctico, el cual posee dos vertientes de investigación: Una basada en la reflexión-acción y otra en el conocimiento personal (Fenstermacher, 1994).

La primera, se fundamenta en los principios de Donal Schön (citado en Fenstermacher, 1994), el cual considera que el Conocimiento Práctico se infiere desde la misma práctica; por ende, se resiste a entender la práctica pedagógica como una mera aplicación técnica del conocimiento. Para sustentar su idea se vale de la "epistemología de la práctica", donde incluye para su comprensión los términos de conocimiento en acción, reflexión en y sobre la acción.

La segunda vertiente está representada por Elbaz; Connelly \& Clandinin (citado en Fenstermacher, 1994), quienes comprenden el Conocimiento Práctico desde las historias y las narrativas docentes.

A través de los estudios en estas dos vertientes, se ha podido obtener que el Conocimiento Práctico, es de carácter situacional, idiosincrático, experiencial, dinámico y producto de infinitos factores (Contextuales, cognitivos, biográficos y experienciales), que en conjunto generan conocimientos distintos a los impartidos en la formación inicial, ocasionando la apertura a procesos de transformación de las teorías formales (Medina, 2006).

Otro término a rescatar, corresponde al de práctica reflexiva, el cual no es un concepto de reciente construcción, sino que se remonta a varias décadas atrás; iniciando su construcción teórica con pensadores griegos como Platón, con su antiguo dualismo; Sócrates, con su método mayéutico y Aristóteles, con su conocimiento reflexivo. Más 
recientemente, se encuentran pensadores como Rousseau y John Dewey; pero quien más desarrolla el concepto de práctica reflexiva es Donald Schön (Domingo, 2008).

Schön $(1987,1998)$, es considerado el pensador más influyente en el Modelo del Aprendizaje del Profesor Reflexivo, en el siglo XX. Los ejes centrales de éste modelo son la experiencia, la práctica y la reflexión en y sobre la acción.

A través de los anteriores autores, se infiere que la visión que ofrecen de conocimiento profesional unido al de práctica reflexiva, concibe al maestro como investigador y a la producción de conocimiento, como un proceso de investigación reflexiva y crítica del análisis de los problemas prácticos.

La reflexión desde ésta perspectiva, es una forma de lograr que los profesores se emancipen, ya que recuperan sus acciones, las hacen conscientes, las evalúan y reconstruyen su práctica; lo que implica un esfuerzo sistemático de análisis de la experiencia, que posibilita hacer investigación (Montero, 2001).

Estas situaciones, conllevan a que el maestro logre la transformación de sus prácticas escolares, fomentando así acciones formadoras, en la que los profesores alcanzan una fundamentación teórica de su actuación (Cabrera, Rodríguez \& Meinardi, 2009).

A continuación, se presenta la estructuración metodológica de la investigación:

\section{Metodología}

La perspectiva fue etnometodológica (Guber, 2001), el enfoque cualitativo (Taylor \& Bogdan, 1986) y de carácter interpretativo (Tezanos, 2004). Por ello, a continuación se explica brevemente, desde los autores la elección de ésta metodología.

La perspectiva fue etnometodológica, porque tiene presente que el conocimiento del mundo surge de las interrelaciones sociales y de las reproducciones de las mismas. Estas interrelaciones están mediadas por el lenguaje, el cual posee dos propiedades: la indixicalidad y la reflexividad. La primera alude a la capacidad de compartir significados comunes y la segunda corresponde a las descripciones sobre la realidad, donde este último código no informa, sino que es práctico y constitutivo de la situación (Guber, 2001).

El carácter reflexivo de la realidad, permite una perspectiva de búsqueda, donde ubica al investigador en una postura intelectual, ya que constantemente trata de dar sentido a su cotidianidad, mediante la explicación y comprensión de sus formas de vida (Guber, 2001).

Teniendo en cuenta lo anterior, se decidió retomar ésta perspectiva porque ubica a la investigadora como un instrumento de investigación, que está en capacidad de describir, explicar y entender el mundo en que vive. Adicionalmente, le permite plasmar la visión en la que paulatinamente fue construyendo la práctica pedagógica y configurar el conocimiento profesional del profesor desde el quehacer.

El enfoque de investigación empleado fue cualitativo, el cual según Taylor \& Bogdan (1986), se caracteriza por indagar los fenómenos sociales, a partir de la comprensión de los mismos. En este enfoque se prima la subjetividad de las personas partícipes en el 
proceso investigativo y los resultados se obtienen principalmente por medio de la observación.

Así mismo, el carácter del trabajo de grado fue interpretativo (Tezanos, 2004), debido a que contribuyó en el reconocimiento de las diversas formas de pensar y actuar de la docente en formación, dada la comprensión de su praxis.

Por otra parte, el estudio se llevó a cabo en cuatro fases: Definición del problema de investigación y los objetivos, obtención de la información, categorización de datos y análisis de resultados. Ver ilustración 1.

\section{FASES DE INVESTIGACIÓN}

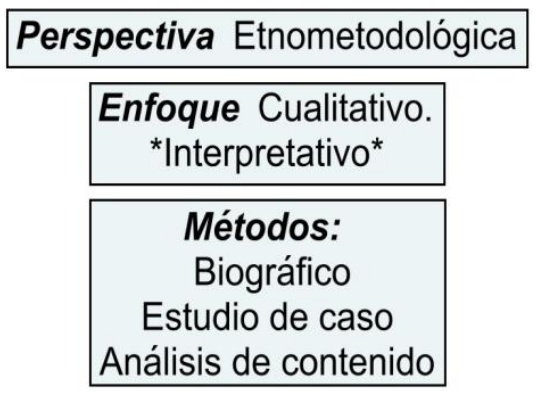

Perspectiva Etnometodológica

Enfoque Cualitativo.

\section{Biográfico}

Análisis de contenido

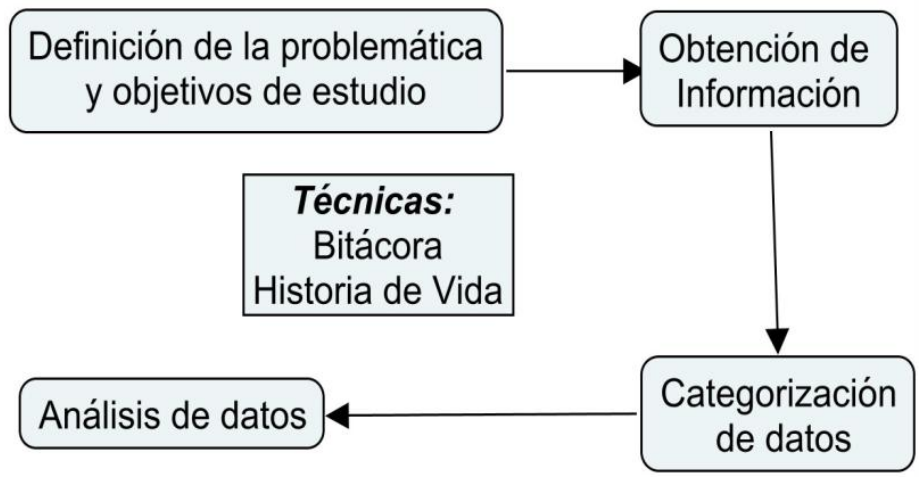

llustración 1: Metodología de Investigación. Tomado de Neira, L (2012:48)

Los métodos empleados fueron diversos, tales como: Método biográfico (Rubio \& Vargas, 1999), análisis de contenido (Abela, 2003) y estudio de caso (Stake, 2007). La elección de estos métodos se debió, a que permitieron profundizar en la comprensión de la observación, la descripción, la interpretación y el análisis de la praxis.

Las técnicas empleadas fueron: Bitácora (Taylor \& Bogdan, 1986) e historias de vida (Rubio \& Vargas, 1999). La primera técnica, ayudó a realizar una reconstrucción narrativa en torno a la praxis. Por ello, se diseñó una bitácora en cuatro columnas: (1) Descripción de la situación, (2) rol del estudiante, (3) rol del practicante y (4) reflexiones por parte de la docente en formación. Estas columnas se dividieron en tres filas (Episodios), que corresponden a las actividades de iniciación, desarrollo y síntesis, planteadas para cada sección de clases. Ver tabla 1.

\begin{tabular}{|c|c|c|c|}
\hline \multicolumn{4}{|c|}{ FORMATO DE BITÁCORA: Práctica Pedagógica } \\
\hline \multicolumn{2}{|l|}{ Fecha: dd/mm/aa } & \multicolumn{2}{|c|}{ Clase №: ------ / hora } \\
\hline Descripción de la Situación & $\begin{array}{ll}\text { Rol del } \\
\text { Estudiante }\end{array}$ & $\begin{array}{lcl}\text { Rol de la } \\
\text { Practicante }\end{array}$ & $\begin{array}{l}\text { Reflexiones de la docente en } \\
\text { formación }\end{array}$ \\
\hline \multicolumn{4}{|l|}{ Episodio 1} \\
\hline \multicolumn{4}{|l|}{ Episodio 2} \\
\hline \multicolumn{4}{|l|}{ Épisodio 3} \\
\hline iones: & & & \\
\hline
\end{tabular}

Tabla 1: Formato de bitácora "Práctica Pedagógica". Tomado de Neira, L (2012:160) 
La segunda técnica empleada, corresponde a la realización de un relato autobiográfico, por parte de la maestra en formación (Rubio \& Vargas, 1999), en relación a sus experiencias de aprendizaje y enseñanza, ya que permite reconocer cómo se ha ido construyendo su identidad profesional.

La sistematización de las reflexiones de la práctica pedagógica, fue realizada según el método de análisis de contenido (Abela, 2003). Atendiendo a ello, en primer instante se identificó las unidades de información, que están consignadas en la cuarta columna de la bitácora y se les asignó un código. Al haber asignado los códigos a las unidades de información, se procedió a ubicar ésta información en el sistema de categorías diseñado. Ver ilustración 2

Posteriormente, se formularon proposiciones agrupadas, que corresponden a la información más representativa en las categorías y las subcategorías. Es prudente aclarar, que no sólo se retomó información de la cuarta columna de la bitácora, también, se seleccionaron unidades de información de las tres primeras columnas, las cuales corresponden a: descripción de la situación, rol de estudiante y rol del profesor.

Ésta acción se debió, a que en el momento de analizar los datos de algunas categorías, éstas brindaron poca o confusa información, ya que al buscar extraer el Conocimiento Práctico, se observó algunas inconsistencias entre los razonamientos y la labor que desarrollo la maestra en el aula.

Respecto al relato autobiográfico, se empleó para su sistematización el método biográfico. Siguiendo los planteamientos de Dante \& Limón (2007), quienes proponen las pautas para el análisis de la narración autobiográfica: Seleccionar las frases significativas, asignarles un código y agruparlas según similitudes de contenido. Estas agrupaciones, son denominadas nodos o tópicos temático - narrativos.

Al analizar cómo se construye el Conocimiento Práctico en el quehacer docente y mediante la reflexión, se debió emplear un sistema de categorías, que integrara no sólo lo parte disciplinar, pedagógica y didáctica. También, reconociera a la docente en formación, como un sujeto de emociones, quien genera juicios y adopta una postura frente a su contexto, posee una historia de vida y tiene un estilo de enseñanza propio. Permitiendo identificar sus habilidades, dificultades para realizar la enseñanza y se le concibiera como un ser, que busca aprender de la experiencia y fortalecer la profesión.

Para tal fin, partiendo de Connelly \& Clandinin (1990), Ángulo (1999) y Medina (2006), se concluyó que el Conocimiento Práctico se ubica dentro de ésta perspectiva. Por ello, fue estructurado un sistema de categorías, que integrara las dos corrientes de investigación del Conocimiento Práctico, identificadas por Fenstermacher (1994).

Como segunda instancia, después de haber establecido las categorías de análisis, éstas fueron modificadas, acorde a las sugerencias hechas por algunos estudiantes de la Maestría en Educación, quienes pertenecen a la Línea de Investigación Conocimiento Profesional del Profesor en Ciencias, de la Universidad Pedagógica Nacional. Dando como resultado, la construcción de un sistema de categorías, para el Conocimiento Práctico, basado principalmente en los planteamientos de Schön (1987), Valbuena (2007), Connelly \& Clandinin (1990). 


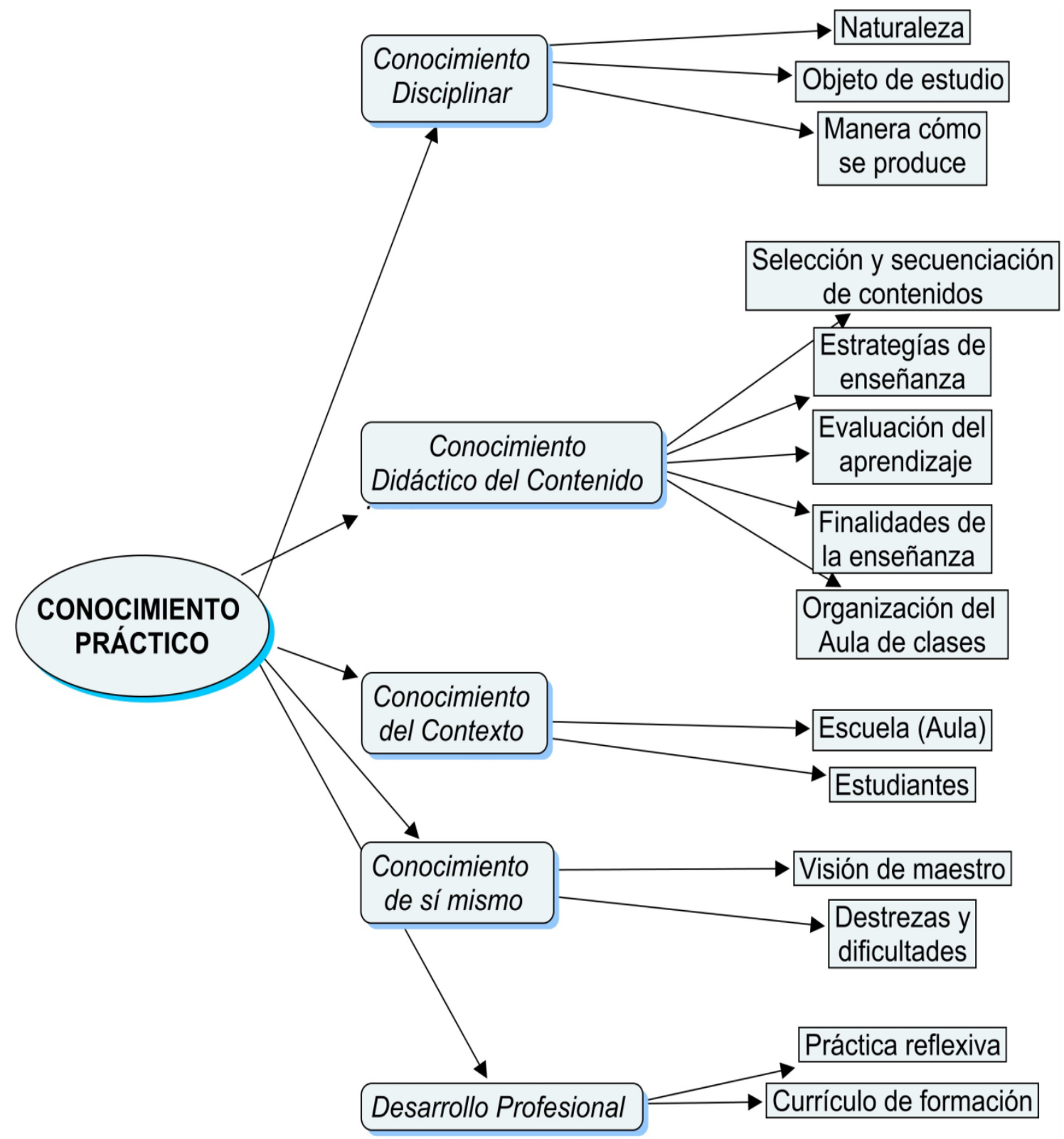

Ilustración 2: Categorías y subcategorías para el Conocimiento Práctico. Tomado de Neira, L (2012:56)

\section{Resultados y discusión:}

En éste apartado se muestra los resultados y análisis de ésta investigación. Por ello, se presenta los datos obtenidos en la bitácora y en el relato autobiográfico; posteriormente, se realiza un corto escrito, que integra la información suministrada por las técnicas y cuál fue su aporte a la construcción de Conocimiento Práctico.

Cabe mencionar, que por extensión del trabajo, no se plasma los resultados en cuanto a los niveles de reflexividad alcanzados en la praxis.

Del mismo modo, debido a que el objeto de estudio es una de las investigadoras, es importante que el lector reconozca la validez de resultados, sin que llegue a pensar en la 
poca credibilidad de ésta investigación, a causa de los matices subjetivos. Pero frente a este suceso, se debe decir que por ello se tuvo en cuenta diversas estrategias de triangulación: Triangulación entre investigadores, triangulación de las fuentes de datos, triangulación metodológica y triangulación teórica (Stake, 2007).

Acorde a lo dicho, la triangulación entre investigadores, se realizó junto con la directora del trabajo de grado.

La triangulación de las fuentes de datos, se hizo para determinar si aquello que se observó, tiene el mismo significado de lo que se informó. Para lograr realizar este tipo de triangulación, se retomó y analizó todas las reflexiones de la práctica pedagógica y algunas descripciones de ésta.

La triangulación teórica correspondió a la utilización de múltiples perspectivas o teorías, para interpretar los datos. Para llevar a cabo esta acción, se realizó un análisis de las preposiciones (Bitácora) y de los tópicos temáticos - narrativo (Relato Autobiográfico), tanto para las subcategorías como para las categorías, desde diversos autores.

Seguidamente, se llevo a cabo la triangulación metodológica, que se caracterizo por contrastar los datos obtenidos en las técnicas. Por lo cual, se empleó la bitácora y el relato autobiográfico, para comprender cómo éstas herramientas aportaron a la construcción del conocimiento práctico.

\subsection{Práctica pedagógica:}

\subsubsection{Conocimiento Disciplinar:}

1.1 .2

1.1.3 Las proposiciones de la maestra en formación tienen relación con lo planteado por Mayr (1988), ya que menciona a la Biología como una ciencia del estudio de lo vivo, la cual se caracteriza por una producción de conocimiento acorde a las particularidades de lo vivo (complejidad). Donde en este proceso de generación de conocimiento biológico, influye el contexto y el concepto de objetividad, que se establece de forma social y que está en constante discusión.

\subsubsection{Así mismo, establece a la ecología como una disciplina, debido a su carácter interdisciplinar y la diferencia del ambientalismo.}

Cl6: 2-05 Es vital esclarecer en el momento de la enseñanza, que los productos de conocimientos de la Biología no solamente se dan a partir de la experimentación, sino también de la observación y la respectiva formulación de teorías

Al examinar de manera general la concepción de la docente en formación, acerca del conocimiento disciplinar de la Biología, se deduce a través de los planteamientos de Valbuena (2007), que las preposiciones estructuradas se ubican en un nivel próximo a la hipótesis de progresión, ya que poseen una visión evolucionada frente a la naturaleza, el objeto y la manera en que se produce el conocimiento biológico. 
Éste resultado se debe, a que en el momento de realizar la praxis la maestra en formación, ya había cursado un espacio académico, denominado "Biología y Conocimiento", en el cual, se busca generar discusiones en torno a las nuevas teorías sobre la epistemología de la Biología.

Al analizar los resultados de ésta categoría, mediante la descripción que realiza Schwab (citado por Estebaranz, 1999), se encuentra que las unidades de información más numerosas, están relacionadas con la estructura sintáctica, que se refiere a las formas de producir y validar el conocimiento.

Cabe resaltar, que las unidades de información correspondientes al conocimiento disciplinar y sus planteamientos no fueron estructurados en profundidad, como tampoco fueron las más representativas en cantidad (4 unidades de información, de 87).

\subsubsection{Conocimiento Didáctico del Contenido:}

1.1.6

1.1.7 Acerca del cómo se enseña la Biología (Ecología), se vislumbra que no se retoma como criterio exclusivo para seleccionar, organizar y secuenciar los contenidos, la fuente disciplinar, sino también, la practicante tuvo en cuenta aspectos relacionados con las características del contexto. Permitiendo, el desarrollo de un currículum flexible y holístico, en el cual buscó promover la adquisición de competencias comunicativas, valores y conocimientos, que puedan ser empleados en la cotidianidad por parte de los alumnos. Cabe resaltar, que aunque reconoció los diferentes tipos de contenidos, sólo evaluó el ámbito disciplinar, el cual estuvo orientado por la perspectiva transitoria, entre medir contenidos y llevar a cabo una evaluación contínua.

Cl5: 1 - 01 Las competencias comunicativas sirven como una herramienta a aplicar en el ámbito social, pero como maestros de Biología, las podemos utilizar como una estrategia para acercar a los estudiantes a una ciencia escolar y encaminar a los futuros estudiantes interesados en esta área de conocimiento, mediante la elaboración de informes de laboratorio, formatos de observación, ensayos, artículos científicos; ya que se parte del reconocimiento de que en la ciencia a lo largo de los años, ha recurrido a las competencias comunicativas, para dar a conocer la construcción de conocimiento.

Para guiar la enseñanza empleó diversas estrategias de enseñanza (videos, comunicación bidireccional, analogías, resúmenes, unidad didáctica, preguntas orientadoras, entre otros). Algunas de estas estrategias corresponden al modelo de Enseñanza para la Comprensión (EpC) y al deseo de desarrollar la competencia comunicativa en los aprendices.

Cl2: 2-03 La comunicación en el aula de clases es fundamental para concretar y mediar actividades, métodos de evaluación y contenidos disciplinares a enseñar a futuro. Donde hay que tener presente, que para éste intercambio de ideas, juegan un papel muy importante las representaciones de poder que posean los partícipes de éste proceso.

Concerniente a la subcategoría de organización, la estipuló desde el aula de clases y desde la institución escolar; evidenciando aspectos del currículum oculto, el cual se enfocó principalmente hacia la adquisición de normas de convivencia y al derecho de una educación contínua, que genere transformación social. 
La información obtenida a lo largo de las diferentes subcategorías, permitió a la docente en formación, reconocer que el conocimiento didáctico del contenido, es un conocimiento contextual, práctico-personal y disciplinar, ya que éste tipo de conocimiento profesional, influye en las decisiones que se toma para transformar los contenidos seleccionados, en un conocimiento enseñable.

Al analizar el conocimiento didáctico de manera general, se evidencia la importancia de la reflexión, para superar la mera racionalidad técnica de ésta categoría, ya que no basta con dominar algunas estrategias didácticas o seleccionar y secuenciar los contenidos, sino también, se requiere examinar los cambios en las dinámicas de enseñanza y aprendizaje.

1.1 .8

1.1.9 Conocimiento del contexto:

1.1 .10

1.1.11 Respecto a la escuela (Aula), la practicante la percibe desde una postura tradicional. Pero, la descripción no sólo se limita a ésta subcategoría, sino también reconoce a los estudiantes desde sus múltiples características.

Cl6: 1 - 02 La institución educativa es vista como un espacio de encierro y control, donde no se observa como un establecimiento para explorar nuevos espacio de aprendizaje, desde el ámbito académico y social.

La información recopilada a través de ésta subcategoría, fue de vital importancia para la maestra, porque le aportó en la estructuración del programa de enseñanza y en la identificación de que la escuela, es una institución social. Por lo cual, ésta influye en otros contexto y a su vez, las dinámicas de otros contextos condicionan el papel de la escuela.

1.1.12

1.1.13 Conocimiento de sí mismo:

1.1.14

1.1.15 Se evidenció las destrezas y dificultades de la docente en formación, las cuales están relacionadas con rasgos de su personalidad y con quehaceres propios del enseñante. Al analizar ésta subcategoría, se encontró que las emociones emergidas en la práctica pedagógica, fueron un elemento de gran importancia, para el desarrollo personal y profesional, debido a que promovían la reflexión.

Cl7: 2-03 Una de las mayores dificultades para enseñar, es poder controlar los problemas de indisciplina, ya que ésta se relaciona con actitudes de irrespeto y violencia física por parte de los estudiantes hacia la docente, como también entre los mismos estudiantes

ClØ3: R - 01 El docente por muchos años se ha visto como transmisor de conocimientos y las mismas instituciones que lo forman han reforzado éste ideal, ya que centran más sus discusiones, sí sus currículos de formación deben estar estructurados hacia el fortalecimiento del dominio disciplinar o el pedagógico y didáctico. Esto ha repercutido en el desconocimiento del enseñante, como un sujeto de conocimiento diverso y como un sujeto político. 
Junto a éstos hallazgos, las apreciaciones acerca de la profesión (colectivo), se enfocan a lo que denomina Marcelo (1995), como una profesión plana, la cual tiene pocos incentivos. También, percibe a la educación, como una labor de todos (familia, estado, escuela), donde el papel del profesor, es orientar la enseñanza de contenidos, por medio del reconocimiento del contexto.

Como último, manifiesta la importancia de la realización de prácticas docentes de autoreconocimiento y de auto-evaluación, porque a través de éstas se genera un crecimiento profesional. Además, permiten percibir al profesor, como un sujeto de emociones y no meramente cognoscente.

El planteamiento de introspección docente, también lo argumenta desde la postura de fomentar una cualificación continua, en los primeros años de vida profesional, ya que las primeras experiencias como profesor, son las que impregnan de concepciones las futuras prácticas.

\section{Desarrollo profesional:}

Éste concepto lo entrelaza con el currículo de formación docente y éste a su vez con la práctica reflexiva. Ésta relación le aporta elementos para comprender, que las instituciones de educación superior docente, deben brindar una formación integral, tanto en los diversos tipos de conocimientos y métodos de investigación. Sumando a esto, deben incentivar la necesidad de una cualificación permanente, en función de las actuales demandas de la sociedad.

Cl8: 2-02 Es necesario que los currículos de formación docente, estén orientados hacia el desarrollo del conocimiento profesional en los egresados, acorde a las necesidades del contexto. Haciendo especial énfasis en el desarrollo de habilidades investigativas.

Cl5: 1-07 Considero que en la formación inicial, al llevar a cabo la práctica pedagógica, la reflexión sobre la acción, es la labor más importante para fortalecer el quehacer, ya que en ocasiones no se ha adquirido el hábito de aprender a reflexionar en la acción, debido a las múltiples labores al enseñar.

\section{Relato autobiográfico:}

En la narración acerca de las experiencias de vida en instituciones escolares, se identificó tres grandes momentos, como lo son: La etapa del preescolar, la etapa de la educación media y básica, y la etapa de la educación superior (Pregrado). En las cuales, da a conocer los hechos y personas, que marcaron o encaminaron su decisión frente al estilo de vida seleccionado (Docencia).

En primer momento, la maestra en formación muestra información concerniente a su personalidad, en donde descubre elementos relacionados con la posibilidad de enseñar a otros.

La segunda etapa, está relacionada con la educación media y básica recibida, donde destaca una formación religiosa, en valores y tradicional. En éste período revela, que muchos profesores le dejaron una huella, a causa de su estilo de enseñanza, lo cual le 
permitió identificar problemas relacionados con el aprendizaje y a explorar qué habilidades o conocimientos le llamaban la atención.

Como último y tercer momento, al ingresar a la educación superior, se evidenció elementos para comprender cómo se fue construyendo su identidad profesional, la cual estuvo cimentada a través de dilemas.

“...Instituto de María, donde sólo realicé primero y segundo de primaria, donde resalto que su enseñanza era principalmente conductista y personalizada, a causa de la poca cantidad de estudiantes por cada salón...ingrese al Colegio Medalla Milagrosa, que estaba bajo concesión de la comunidad religiosa de las Hermanas de Marie Pausepin, donde logre culminar mis estudios de básica primaria...entre al Colegio la Presentación que también estaba coordinada por ésta comunidad religiosa, donde realice mi bachillerato con énfasis en contabilidad..." R(D.f) - 04

“...el otro docente que me dio Ciencias Naturales, no le interesaba tanto el aprendizaje de contenidos biológicos, sino que creciéramos en valores relacionados con el área o la vida en sociedad; viéndose reflejada ésta postura en las constantes lecturas dejadas...En algunas ocasiones éstos escritos eran retomados como excusa para ver los contenidos a enseñar, pero casi siempre al enseñante sólo le interesaba que le relatáramos nuestras apreciaciones éticas y cuáles las llevaríamos a nuestra cotidianidad..."R(D.f) - 08

“... espacio curricular, denominado Enseñanza de la Biología, al cual le tome gran aprecio y valor, debido a que el profesor que daba esa materia me recordaba a mi abuelito...éste maestro citó en clase al cantautor Diomedes Díaz, en su estrofa: "Por eso Rafael Santos yo quiero/dejarte dicho en esta canción/que si te inspira ser zapatero/sólo quiero que seas el mejor/porque de nada sirve el doctor/si es el ejemplo malo del pueblo". Lo que ocasiono en mí ser, el meditar que si ya no había logrado ingresar a la Universidad que deseaba y estudiar la carrera que quería, ahora en adelante debía esforzarme por ser una excelente maestra. Es curioso resaltar, que al final de ese año, surgió de la nada un inmenso amor por la docencia, que aún no me logro explicar y en tan corto tiempo." R(D.f) - 19

Relato autobiográfico vs. Bitácora:

"Construcción del Conocimiento Práctico":

Al haber relatado muy brevemente lo hallado, ahora se analiza conjuntamente los resultados obtenidos en las dos técnicas, donde se evidenció que la docente en formación, puso en juego los diferentes tipos de conocimiento profesional y los entretejió por medio de la unidad didáctica, ya que en ella plasmó su particular forma de concebir la educación y el conocimiento aprendido a través de la formación. Donde las decisiones tomadas se fueron moldeando, por las dinámicas con el contexto escolar.

De otro lado, entre el accionar docente, el plan de enseñanza y la postura frente educación, se presentaron contrasentidos, ya que se determinó que en situaciones problemáticas, la maestra en formación recurría a medidas tradicionales y en otras no era consciente de las prácticas contradictorias. Estos contrasentidos se presentaron 
principalmente en situaciones emotivas (estrés, felicidad, filiación afectiva con los estudiantes, entre otras), las cuales ocasionaron que incrementara la significatividad de la experiencia.

A partir de éstos, surge el planteamiento de que para comprender el Conocimiento Práctico, se necesita el poder diferenciar entre "el pensamiento real" y "el pensamiento ideal", pues éste último representa un velo o sesgo para el investigador al momento de analizar el Conocimiento Práctico docente.

Lo que se denomina como "pensamiento real", es la concordancia entre la postura frente a los modelos de enseñanza-aprendizaje y el accionar. Entre tanto, el "pensamiento ideal", es la postura frente a la enseñanza-aprendizaje, desde lo que "debería ser" el quehacer del maestro, ya que las acciones no concuerdan con lo expresado, acerca del proceso educativo (Neira, 2012).

Recapitulando lo dicho, se identificó que el Conocimiento Práctico, es de carácter contextual, dinámico, idiosincrático, biográfico y práctico, donde éstas características ya han sido descritas desde diversos autores que trabajan ésta temática (Medina, 2006; Connelly \& Clandinin, 1990; Montero, 2001). Con respecto a esto, se añade el carácter contradictorio-emotivo, tradicional, complejo y reflexivo; donde éstas características serán esclarecidas más adelante.

Igualmente, se deduce que el análisis de la praxis, permite identificar principalmente los conocimientos explícitos. Esto se debe a que al planificar la enseñanza, se tuvo la necesidad de esclarecer qué conocimiento profesional quería integrar y cuáles serían las metas a alcanzar por parte de la practicante.

Por otra parte, el relato autobiográfico permitió evidenciar cómo las vivencias, fueron construyendo tanto la identidad personal, como profesional y desde ésta mirada retrospectiva ayudó a descubrir la génesis y evolución del Conocimiento Práctico docente, ya que mediante la interpretación del conocimiento y su relación con experiencias del pasado, dio sentido al accionar educativo.

Cabe resaltar, que en el escrito autobiográfico se mostró esencialmente un conocimiento tácito, porque muchas de las vivencias generaron concepciones, que hasta ahora la docente en formación reconoce. Ver tabla 2.

\begin{tabular}{|l|l|}
\hline Pasado & Presente \\
\hline $\begin{array}{l}\text { La formación religiosa y tradicional } \\
\text { recibida. }\end{array}$ & $\begin{array}{l}\text {-El fin de la educación es forma para la } \\
\text { vida cotidiana y en valores. } \\
\text {-Prácticas contradictorias y rígidas }\end{array}$ \\
\hline $\begin{array}{l}\text { Auto-reconocimiento. } \\
\text {-Socialización con niños } \\
\text {-Habilidad en manualidades } \\
\text {-Explorar y conocer el mundo natural }\end{array}$ & $\begin{array}{l}\text {-Elementos para apropiarse de una } \\
\text { identidad docente. }\end{array}$ \\
\hline Formación inicial docente. & -Fomentar actividades artísticas \\
& $\begin{array}{l}\text {-Teorías pedagógicas y didácticas Vs. } \\
\text { Necesidades del contexto. } \\
\text {-Políticas educativas Vs. Contexto. }\end{array}$ \\
\hline Crisis de la identidad docente. & $\begin{array}{l}\text {-La necesidad de un docente con } \\
\text { identidad, que sea crítico-reflexivo, }\end{array}$ \\
\hline
\end{tabular}


investigador: Sujeto de conocimientos.

Tabla 2: Relación entre los datos de la bitácora y el relato autobiográfico. Tomado de Neira, L

(2012:125)

Frente a éstos resultados, Gimeno \& Pérez (1988), postulan que el Conocimiento Práctico se construye progresivamente a través de la socialización, en donde las instituciones sociales, como la escuela, impregnan de modelos de enseñanza a los sujetos, los cuales los adquieren por medio de la observación y la exposición prolongada de esquemas de comportamiento de la vida escolar.

Al observar éste suceso y al analizar de manera integrada los resultados, se postuló que el Conocimiento Práctico, es un pensamiento conservador, que se alimenta por la tradición social y las posturas institucionalizadas. Éste conocimiento logra ser cambiante, a causa de la interacción con el medio y su reflexionar.

Del mismo modo, se propone que se debe entender como un pensamiento complejo, ya que se encuentra condicionado por el contexto; por las concepciones éticas, morales o políticas, adquiridas y transformadas a través de las vivencias; por la historia personal; por las características del sujeto y está ligado a la práctica. Es decir, es un conocimiento completo, por su rica interacción de factores.

Para culminar, se postula que para comprender el Conocimiento Práctico, se requiere tanto el análisis de las narraciones de vida como de la práctica pedagógica, ya que permiten vislumbrar el conocimiento tácito y el consciente, en los cuales se evidencia la necesidad de reflexionar sobre las experiencias, para poder expresar el conocimiento construido y entender el sentido de la actuación docente.

De acuerdo con lo anterior, en el Conocimiento Práctico, la reflexión, es un elemento vital, porque ayuda a reconstruir éste conocimiento, por medio del examen sistémico de la propia experiencia, posibilitando que el docente aprenda de ella y disminuya las rutinas de acción. Así mismo, la práctica reflexiva permite el carácter dinámico de éste conocimiento, porque suscita valoraciones acerca del quehacer, que a su vez aporta en su transformación.

\section{Conclusiones:}

En éste apartado se encuentra las conclusiones concernientes a los objetivos y al aspecto metodológico. Donde en primer instante, se concluye que el sistema de categorías para Conocimiento Práctico, integró los diversos contenidos de la formación profesional y reconoció a la docente, como un sujeto de emociones, la cual posee una historia de vida y un estilo de enseñanza propio.

Cabe resaltar, que al estructurar las categorías, a partir del análisis documental y del proceso de sistematización de datos, surgió una categoría emergente, denominada "Desarrollo profesional". Ésta categoría ubica al enseñante, como un individuo productor de conocimiento, con relación a la práctica reflexiva y a los currículos de formación docente.

Al categorizar la información suministrada por las técnicas, se evidencia la necesidad de triangular las descripciones de la práctica, sus reflexiones y entenderlas a la luz del relato 
de vida. Debido a que el Conocimiento Práctico, está ligado a la práctica, a la subjetividad, a la formación docente recibida y a su respectiva reflexión.

A través del proceso de análisis de la información recopilada en las categorías, se ratificó las características descritas para el Conocimiento Práctico (Medina, 2006; Connelly \& Clandinin, 1990; Montero, 2001) y se halló algunas nuevas particularidades, como lo son: Su carácter contradictorio-emotivo, tradicional, complejo y reflexivo; y que para su análisis se requiere la diferenciación entre "el pensamiento real e ideal".

Frente a estos hallazgos, es posible establecer que el camino hacia la construcción del Conocimiento Práctico está ligado a la práctica reflexiva. Dado que, al esclarecer el significado del qué, dónde y cómo se desarrolla el quehacer docente; permite generar incertidumbres que se moldean y depuran en la práctica pedagógica, originando nuevos conocimientos.

Estos conocimientos no deben ser expuestos a una lucha, por legitimar la producción de conocimiento y el saber, sino deben ser percibidos como una producción de conocimiento, en términos de un contexto y el uso de los mismos, en un espacio específico.

Ahora, al centrar las conclusiones en el desarrollo metodológico, se debe mencionar que para alcanzar el objetivo propuesto, se debió superar diversos retos, que se relacionan con el enfoque cualitativo e intersubjetivo de esta investigación (Reflexión de la propia práctica pedagógica).

Acorde a lo anterior, aparece la subjetividad como un arma de doble filo, que brinda la posibilidad de percibir a la maestra en formación como un sujeto de conocimiento, cuyo rol es el de ser objeto de estudio y sujeto investigador. Por ello, fue indispensable el desarrollo metodológico riguroso y honesto en el diseño de los relatos, ya que en gran medida esta postura condicionaba la validez de resultados.

En relación a las técnicas de historia de vida y bitácora, se determina que el éxito de su aplicación, está condicionado por el adecuado proceso de observación participante y de estimulación del recuerdo; en los cuales es indispensable rescatar un discurso descriptivo de la realidad acorde a los sucesos.

Así mismo, se encuentra que dentro del posicionamiento teórico del Conocimiento Práctico, se privilegia el maestro capaz de producir su propio conocimiento, a causa de que resulta más significativa la experiencia, dejando de lado la mirada tradicional y hegemónica de la epistemología clásica.

\section{Sugerencias}

Después de haber vivenciado el proceso de investigación, es oportuno realizar algunas recomendaciones para próximos estudios. Por ello, es imperante recurrir a diversas fuentes de información, para construir el Conocimiento Práctico, como lo son: Cuestionarios, sesiones de reflexiones orientadas, videos de la práctica pedagógica, entrevistas de los tutores de práctica y análisis del diseño de las actividades educativas, 
entre otros. Donde al seleccionar los instrumentos, hay que tener como criterio, el favorecimiento de los procesos de verbalización y reflexión de las situaciones.

Para llevar a cabo, la práctica reflexiva, resulta insuficiente la experiencia en el quehacer, sino se ha entendido éste concepto como un proceso metacognitivo, el cual en las primeras etapas de apropiación, el sujeto necesita aprender a reflexionar en y sobre la acción, porque son múltiples los sucesos a reflexionar. Por consiguiente, se debe adquirir paulatinamente la capacidad de seleccionar las vivencias, que generen nuevos conocimientos.

Para seleccionar las experiencias más significativas, que sirvan de fundamento para reflexionar sobre y para la acción, se sugiere recurrir a preguntas orientadoras, ya que las mismas fortalecen el desarrollo de las reflexiones.

Estas reflexiones deben ser percibidas en términos de un aprendizaje, porque de ésta manera, es posible producir nuevos significados de la realidad, los cuales sirvan como elemento para trasegar por el camino del aprendizaje.

Otro aspecto, es que la práctica reflexiva cuenta con escasos estudios, que sirvan de antecedentes para determinar los métodos y las técnicas a seleccionar, acorde a los objetivos estipulados para cada investigación. Por tanto, se aconseja que las metodologías estructuradas, tenga como fundamento, el reconocer al maestro desde las narrativas y a partir de las historias de vida, porque a través de éstas se rescata la subjetividad y se construye la identidad.

Así mismo, se recomienda identificar las emociones y los inconvenientes que surgen el quehacer, ya que son un motor para potencializar las reflexiones.

\section{BIBLIOGRAFÍA}

Abela, J. (2003). Las técnicas de Análisis de Contenido: Una Revisión Actualizada. Extraída el 14 julio, 2010, de http://www.fundacion-centra.org/pdfs/S200103.PDF.

Ángulo, F. (1999). De la investigación sobre la enseñanza al conocimiento docente. En A. Pérez, J. Barquín \& F. Ángelo (Ed.), Desarrollos profesional del docente. Política, investigación y práctica (pp. 261-319). Madrid: AKALTextos.

Cabrera, J; Rodríguez, I \& Meinardi, E. (2009). Los Procesos Metarreflexivos en la Formación Inicial del Profesorado. RevistaTecné, Episteme y Didaxis: TED, No. Extraordinario, 986-991

Connelly, F \& Clandinin, J. (1990). Stories of Experience and Narrative Inquiry. En Educational Researcher. 19 (5), 2-14.

Dante, D \& Limón, G. (2007). Relato autobiográfico e identidad personal: Un modelo de análisis narrativo. Revista de Antropología Iberoamericana, 2 (2), 232 - 275. Extraído el 22 Abril, 2011 del sitio web de Organización de Estados Iberoamericanos http://www.oei.es/index.php y luego www.aibr.org 
Domingo, Á. (2008). La Práctica Reflexiva en la Formación Inicial de Maestros/as: Evaluación de un Modelo. Tesis Doctoral. Facultad de Educación. Departamento de Educación Universitat Internacional de Cataluña. Barcelona. Extraído el 20 de junio, 2009 del sitio Web de Universitat Internacional de Cataluña http://www.uic.es/ y luego http://www.tesisenxarxa.net/tdx-0310109.../index.html

Estebaranz, A. (1999). Didáctica e innovación curricular. Sevilla: Universidad de Sevilla.

Fenstermacher, G. (1994). The knower and the known: The nature of knowledge in research on teaching. Review of research in education, 20,3-36

Marcelo, C. (1995). Formación del Profesorado para el cambio educativo. EUB: Barcelona.

Gimeno, J \&Pérez, A. (1988). Pensamiento y acción en el profesor: De los estudios sobre la planificación al pensamiento práctico. Revista Infancia y Aprendizaje, 42, 37-63.

Guber, R. (2001). La Etnografía Método, campo y reflexividad. Bogotá: Norma.

Imbernón, F. (1998). La formación y el desarrollo profesional del profesorado. Cuarta edición. Barcelona: Graó.

Mayr, E. (1998). Así es la biología. Barcelona: Debate. pgs 8-288

Medina, J. (2006). La Profesión Docente y la Construcción del Conocimiento Profesional. Buenos Aires: Magisterio del Río de la Plata.

Montero, L. (2001). Aprender A enseñar: La Construcción del Conocimiento Profesional. En L. Montero (Ed.), La Construcción del Conocimiento Profesional Docente (pp. 133219). Argentina: Homo Sapiens Ediciones.

Neira, L (2009). Desarrollo de las competencias comunicativas a partir de la implementación de una unidad didáctica de ecología y el modelo enseñanza para la compresión. Documento de circulación interna: Universidad Pedagógica Nacional, Facultad de Ciencia y Tecnología. Departamento de Biología.

Neira, L (2012). Aportes a la construcción del conocimiento práctico, como docente en formación de biología, a partir de mi práctica reflexiva de la práctica pedagógica. Trabajo de grado para Optar el Título de Licenciada en Biología. Departamento de Biología. Facultad de Ciencia y Tecnología. Universidad Pedagógica Nacional. Bogotá. Colombia.

Perrenoud, P. (2001). En Desarrollar la Práctica Reflexiva en el Oficio de enseñar. Barcelona: Ed. GRAO.

Estatuto Docente Colombiano: Decreto 1278. (2002). Artículo 7. Ingreso al servicio educativo estatal. En Decreto 1278 de Junio 19 de 2002 "Estatuto de Profesionalización Docente". Extraído el 3 de noviembre, 2009 del sitio Web de Ministerio de Educación Nacional: $\quad$ http://www.mineducacion.gov.co/1621/w3-channel.html y luego http://www.mineducacion.gov.co/1621/article-86102.html. 
Rubio, M \& Vargas, J. (1999). El Análisis de la Realidad, en la Intervención Social. Métodos y Técnicas de Investigación. Madrid: CCS.

Schön, D. (1987). La Formación de Profesionales Reflexivos: Hacia un Nuevo Diseño de la Enseñanza y el Aprendizaje en las profesiones. España: Ed. Paidos.

Schön, D. (1987). La Formación de Profesionales Reflexivos: Hacia un Nuevo Diseño de la Enseñanza y el Aprendizaje en las profesiones. España: Ed. Paidos.

Schön, D. (1998). Profesional Reflexivo: Cómo Piensas los Profesionales cuando Actúan.Barcelona: Paidós.

Soto, C. (2006). Precongreso Regional: La profesión Docente. Revista Educación y Pedagogía, Marzo, Sepanata, 9-11

Stake, R. (2007). Investigación como Estudio de Casos. Madrid: Morata. pgs. 11-117

Tardif, M. (2004). Los saberes del docente y su desarrollo profesional. Madrid: Narcea, traducción de Pablo Manzano.

Taylor, S \& Bogdan, R. (1986). Introducción a los Métodos Cualitativos de Investigación. Buenos Aires: Paidós.

Tezanos, Araceli de. (2004). Una Etnografía de la Etnografía. Bogotá: Ed. Antropos.

Valbuena, E. (2007). El Conocimiento Didáctico Del Contenido Biológico: Estudio De Las Concepciones Disciplinares $Y$ Didácticas De Futuros Docentes De La Universidad Pedagógica Nacional (Colombia). Tesis para Optar de Título de Doctor. Departamento de Didáctica de las Ciencias Experimentales. Facultad de Educación. Universidad Complutense De Madrid. Madrid. España.

Viciana, J; Delgado, M \& Del Villar, F. (1997). El análisis de los niveles de reflexión en el discurso. Su aplicación desde la perspectiva del formador de formadores al campo de la Educación Física y al Entrenamiento Deportivo, Revista Motricidad, 3, 151-175. Extraído el 10 Enero, 2011 de http://journals.apa.org/prevention/volume5/pre0050002a.html

Viscaíno, A. (2008). El conocimiento Práctico en la Formación Docente: Una Construcción Histórica entre Actores e Instituciones. Revista Iberoamericana de Educación, 46 de abril, $1-25$. 